ELECTRONIC RESEARCH ANNOUNCEMENTS OF THE AMERICAN MATHEMATICAL SOCIETY

Volume 8, Pages 20-28 (September 6, 2002)

S $1079-6762(02) 00102-6$

\title{
REGULAR NEIGHBOURHOODS AND CANONICAL DECOMPOSITIONS FOR GROUPS
}

\author{
PETER SCOTT AND GADDE A. SWARUP
}

(Communicated by Walter Neumann)

\begin{abstract}
We find canonical decompositions for finitely presented groups which essentially specialise to the classical JSJ-decomposition when restricted to the fundamental groups of Haken manifolds. The decompositions that we obtain are invariant under automorphisms of the group. A crucial new ingredient is the concept of a regular neighbourhood of a family of almost invariant subsets of a group. An almost invariant set is an analogue of an immersion.
\end{abstract}

This article is an announcement of work whose details will appear later. In this work, we study analogues for groups of the classical JSJ-decomposition (see Jaco and Shalen 9, Johannson [10 and Waldhausen 19]) for orientable Haken 3 -manifolds. The orientability restriction is not essential but it will simplify our discussions. Previous work in this field has been carried out by Kropholler [11, Sela [18, Rips and Sela [13, Bowditch [2], 3], Dunwoody and Sageev [5], and Fujiwara and Papasoglu [8], but none of these results yields the classical JSJdecomposition when restricted to the fundamental group of an orientable Haken manifold. In our work, we give a new approach to this subject, and we give decompositions for finitely presented groups which essentially specialise to the classical JSJ-decomposition when restricted to the fundamental groups of Haken manifolds. An important feature of our approach is that the decompositions we obtain are unique and are invariant under automorphisms of the group. In previous work such strong uniqueness results were only found for decompositions of word hyperbolic groups. Most of the results of the previous authors for virtually polycyclic groups can be deduced from our work. But our arguments use some of the results of these authors, particularly those of Bowditch. In addition, we use the important work of Dunwoody and Roller in 4. Our work also yields some extensions of the results on the Algebraic Annulus and Torus Theorems in [16, 2], and 6]. It should be remarked that even though we obtain canonical decompositions for all finitely presented groups, these decompositions are often trivial. This is analogous to the fact that any finitely generated group possesses a free product decomposition, but this decomposition is trivial whenever the given group is freely indecomposable.

Our ideas are based on an algebraic generalisation of the Enclosing Property of the classical JSJ-decomposition. This property can be described briefly as follows.

Received by the editors May 1, 2002, and, in revised form, July 23, 2002.

2000 Mathematics Subject Classification. Primary 20E34; Secondary 57N10, 57M07.

Key words and phrases. Graph of groups, almost invariant set, characteristic submanifold.

First author partially supported by NSF grants DMS 034681 and 9626537. 
For an orientable Haken 3-manifold $M$, Jaco and Shalen 9] and Johannson [10] proved that there is a family $\mathcal{T}$ of disjoint essential annuli and tori embedded in $M$, unique up to isotopy, and with the following properties. The manifolds obtained by cutting $M$ along $\mathcal{T}$ are simple or are Seifert fibre spaces or $I$-bundles over surfaces. The Seifert and $I$-bundle pieces of $M$ are said to be characteristic, and any essential map of the annulus or torus into $M$ can be properly homotoped to lie in a characteristic piece. This is called the Enclosing Property. The characteristic submanifold $V(M)$ of $M$ consists essentially of the union of the characteristic pieces of the manifold obtained from $M$ by cutting along $\mathcal{T}$. The fundamental group $G$ of $M$ is the fundamental group of a graph $\Gamma$ of groups, whose underlying graph is dual to the frontier of $V(M)$. Thus the edge groups of $\Gamma$ are all isomorphic to $\mathbb{Z}$ or $\mathbb{Z} \times \mathbb{Z}$, and the vertex groups are the fundamental groups of simple manifolds or of Seifert fibre spaces or of surfaces. The uniqueness up to isotopy of the splitting family $\mathcal{T}$ implies that $\Gamma$ is unique. Further, the Enclosing Property implies that any subgroup of $G$ which is represented by an essential annulus or torus in $M$ is conjugate into a characteristic vertex group.

All the previous algebraic analogues of the topological JSJ-decomposition consist of producing a graph of groups structure $\Gamma$ for a given group $G$ with some "characteristic" vertices. The algebraic analogue of the topological Enclosing Property which was used is the property that certain "essential" subgroups of $G$ must be conjugate into one of the characteristic vertex groups of $\Gamma$, but the precise meaning of the word "essential" varies depending on the authors. For example, when trying to describe all splittings of a group $G$ over infinite cyclic subgroups, previous authors produced a decomposition with infinite cyclic edge groups, such that if $G$ splits over an infinite cyclic subgroup $H$, then $H$ is conjugate into a characteristic vertex group. These results are often referred to vaguely but collectively as the JSJdecomposition of a finitely presented group. While they are commonly regarded as being an algebraic analogue of the topological JSJ theory, none of them recovers the topological result when applied to the fundamental group of an orientable Haken 3-manifold. One reason for this is the fact that the Enclosing Property of the characteristic submanifold $V(M)$ is stronger than the algebraic analogue discussed above.

Our results also yield a graph of groups structure $\Gamma$ for a given group $G$ with some "characteristic" vertices, but our algebraic generalisation of the topological Enclosing Property corresponds more closely to the topological situation. Also when trying to describe all splittings of a group $G$ over infinite cyclic subgroups, the decomposition we produce may have edge groups which are not infinite cyclic. This reflects the topological situation better.

Here is an introduction to our ideas. As mentioned before, the Enclosing Property of the characteristic submanifold $V(M)$ of a Haken 3-manifold $M$ means that $V(M)$ contains a representative of every homotopy class of an essential annulus or torus in $M$. We will say that it encloses every essential annulus and torus in $M$. In our work, we introduce a natural algebraic analogue of enclosing. If we restrict attention to embedded surfaces, this analogue is simple to explain. First recall the graph of groups structure $\Gamma$ for $G=\pi_{1}(M)$, whose underlying graph is dual to the frontier $\operatorname{fr}(V(M))$ of $V(M)$. Let $F$ be an embedded essential annulus or torus in $V(M)$, so that $F$ determines a splitting of $G$, and let $\Gamma_{F}$ denote the graph of groups structure for $G$, whose underlying graph is dual to $\operatorname{fr}(V(M)) \cup F$. Thus $\Gamma_{F}$ has one 
more edge than $\Gamma$, and this extra edge corresponds to $F$. Collapsing this extra edge of $\Gamma_{F}$ yields $\Gamma$ again. Now let $G$ be any group, let $\Gamma$ be a graph of groups structure for $G$, and let $\sigma$ be a splitting of $G$. We say that $\sigma$ is enclosed by a vertex $v$ of $\Gamma$, if there is a graph of groups structure $\Gamma_{\sigma}$ for $G$, with an edge $e$ which determines the splitting $\sigma$, such that collapsing the edge $e$ yields $\Gamma$, and $v$ is the image of $e$. We emphasise that the condition that $\sigma$ is enclosed by the vertex $v$ is in general stronger than the condition that the edge group of $\sigma$ is conjugate into the vertex group of $v$. This is particularly clear if $\sigma$ is a free product decomposition of $G$, as then the edge group of $\sigma$ is trivial.

An important observation is that $V(M)$ is closely related to a regular neighbourhood of some (finite) union of essential annuli and tori in $M$. In some cases, one can choose a finite family of essential annuli and tori in $V(M)$ so that $V(M)$ is a regular neighbourhood of their union. More usually, $V(M)$ can be obtained from such a regular neighbourhood by adding solid tori to compressible torus boundary components. In particular, except for a few special cases, $V(M)$ is minimal, up to isotopy, among incompressible submanifolds of $M$ which enclose every essential annulus and torus in $M$. Thus it seems natural to think of $V(M)$ as a regular neighbourhood of all the essential annuli and tori in $M$. The peripheral pieces of the characteristic submanifold can be thought of as a regular neighbourhood of all the essential annuli only. Our main results can be thought of as algebraic versions of these statements. In order to explain our ideas further, we need to discuss the algebraic analogues of immersed annuli and tori and the algebraic analogue of a regular neighbourhood.

An analogue of a $\pi_{1}$-injective immersion in codimension 1 has been studied by group theorists for some time. If $H$ denotes the image in $G$ of the fundamental group of the codimension-1 manifold, this analogue is a subset of $G$ called a $H$ almost invariant set or an almost invariant set over $H$. Any $\pi_{1}$-injective immersion in codimension 1 has a $H$-almost invariant set associated to it in a natural way. In particular, this applies to any splitting of $G$. Further, there is a natural idea of what it means for an almost invariant subset of a group $G$ to be enclosed by a vertex of a graph of groups decomposition for $G$, which generalises the idea of enclosing a splitting.

We briefly recall the basic properties of almost invariant sets. We say that two sets are almost equal if their symmetric difference is finite. A subset $X$ of $G$ is $H$ almost invariant if $H X=X$, and for every $g \in G, H \backslash X$ and $(H \backslash X) g$ are almost equal. A $H$-almost invariant subset $X$ of $G$ is nontrivial if $H \backslash X$ is infinite and has infinite complement in $H \backslash G$. A $H$-almost invariant subset $X$ of $G$ is equivalent to a $K$-almost invariant subset $Y$ if the images of $X \cap Y^{*}$ and $X^{*} \cap Y$ in $H \backslash G$ are both finite. This relation is an equivalence relation, and is very natural. For example, the $H$-almost invariant sets associated to homotopic $\pi_{1}$-injective immersions in codimension 1 are equivalent. These ideas are closely related to the theory of ends of spaces and groups. In particular, $G$ has a nontrivial $H$-almost invariant subset if and only if the pair $(G, H)$ has more than one end. The appropriate notions of intersection and disjointness for such sets were introduced by Scott in [14], and were further developed in [15]. In [14], Scott defined the intersection number of two nontrivial almost invariant subsets of a group and showed it was symmetric. Further his definition generalises the natural idea of intersection number of curves on a surface, and the intersection number of surfaces in a 3-manifold introduced in 
[7. In [15, the main results, Theorems 2.5 and 2.8, were algebraic analogues of the facts that curves on a surface with intersection number zero can be homotoped to be disjoint, and that a curve with self-intersection number zero can be homotoped to cover an embedding. These results do strongly suggest that an almost invariant set is the appropriate analogue of an immersion.

The key new idea of our work is an algebraic version of regular neighbourhood theory. This part of our work applies to arbitrary finitely generated groups. We define an algebraic regular neighbourhood of a family of nontrivial almost invariant subsets of a group $G$. The idea of a regular neighbourhood of two splittings was developed by Fujiwara and Papasoglu [8] in special cases (it can be seen that their enclosing technique yields the same result as our regular neighbourhood construction in these cases), but from our point of view enclosing almost invariant sets is more basic. In our algebraic construction of regular neighbourhoods, as well as several other techniques, we have greatly benefited from the two papers of Bowditch [2] 3]. Bowditch's use of pretrees showed us how to enclose almost invariant sets under very general conditions. In the case of word hyperbolic groups, Bowditch [2] was effectively the first to enclose such sets although he does not use this terminology.

In order to explain the idea of an algebraic regular neighbourhood, we return to the characteristic submanifold $V(M)$ of a 3-manifold $M$ and the graph of groups decomposition $\Gamma$ of $G=\pi_{1}(M)$, whose underlying graph is dual to the frontier $\operatorname{fr}(V(M))$ of $V(M)$. Note that $\Gamma$ is naturally a bipartite graph, because its vertices correspond to components of $V(M)$ or of $M-V(M)$, and each edge of $\Gamma$ joins vertices of distinct types. The vertices which correspond to $V(M)$ will be called $V_{0}$-vertices and the vertices which correspond to $M-V(M)$ will be called $V_{1^{-}}$ vertices. Here are two properties of $V(M)$, which have algebraic analogues. The first is the Enclosing Property, which says that any essential annulus or torus in $M$ is enclosed by $V(M)$. The second is that if $F$ is any embedded essential surface in $M$, not necessarily an annulus or torus, and if $F$ has intersection number zero with every essential annulus and torus in $M$, then $F$ is homotopic into $M-V(M)$. These conditions are not sufficient to characterise $V(M)$ up to isotopy, but they do contain much of the information needed for such a characterisation. The algebraic analogue of the Enclosing Property is that the almost invariant subsets of $G$ which correspond to essential annuli or tori are enclosed by the $V_{0}$-vertices of $\Gamma$. The algebraic analogue of the second property is that the splitting associated to $F$ is enclosed by a $V_{1}$-vertex of $\Gamma$.

Now let $G$ be a finitely generated group with a family of subgroups $\left\{H_{\lambda}\right\}_{\lambda \in \Lambda}$. For each $\lambda \in \Lambda$, let $X_{\lambda}$ denote a nontrivial $H_{\lambda}$-almost invariant subset of $G$. Then our algebraic regular neighbourhood of the $X_{\lambda}$ 's in $G$ will be a bipartite graph of groups structure $\Gamma$ for $G$ such that the $V_{0}$-vertices of $\Gamma$ enclose the $X_{\lambda}$ 's, and splittings of $G$ which have intersection number zero with each $X_{\lambda}$ are enclosed by the $V_{1}$-vertices of $\Gamma$. In addition, we need to insist that $\Gamma$ is minimal in order to have any uniqueness results. There is one further technical condition which we need to impose, but we will not describe it here.

Our main result on regular neighbourhoods is the following.

Theorem 1. Let $G$ be a finitely generated group with a family of subgroups $\left\{H_{\lambda}\right\}_{\lambda \in \Lambda}$. For each $\lambda \in \Lambda$, let $X_{\lambda}$ denote a nontrivial $H_{\lambda}$-almost invariant subset of $G$.

(1) If an algebraic regular neighbourhood of the $X_{\lambda}$ 's in $G$ exists, then it is unique. 
(2) If $\Lambda$ is finite, and each $H_{\lambda}$ is finitely generated, then there is an algebraic regular neighbourhood of the $X_{\lambda}$ 's in $G$.

In case (2), every $V_{0}$-vertex group of the regular neighbourhood is finitely generated. However, the $V_{1}$-vertex groups and the edge groups need not be finitely generated. This is why we allow subgroups $H_{\lambda}$ which are not finitely generated in our definition of an algebraic regular neighbourhood, and this turns out to be an important aspect of the theory. Surprisingly, case (2) of the above theorem remains true if we allow the $H_{\lambda}$ 's not to be finitely generated, so long as we insist that whenever this occurs, $X_{\lambda}$ is associated to a splitting.

Now we discuss how we apply this theory to produce our new decompositions of finitely presented groups. It is simpler to restrict attention to one-ended groups. In addition we need to restrict our attention to finitely presented groups because we use certain accessibility results, for example the main result of Bestvina and Feighn in [1]. As Fuchsian groups play an important role in the results, we should explain that we use the term to include not only discrete groups of isometries of the hyperbolic plane, but also to include discrete groups of isometries of the Euclidean plane. The extra groups included are all virtually $\mathbb{Z} \times \mathbb{Z}$.

Before stating our results, we need a little terminology to describe special types of vertex in a graph of groups $\Gamma$. A vertex $v$ of $\Gamma$ is of isolated type if it has valence 2 , is not the sole vertex of a loop, and the two edges incident to $v$ carry groups which map to the vertex group $G(v)$ of $v$ by an isomorphism. A vertex $v$ of $\Gamma$ is of finite-by-Fuchsian type if $G(v)$ is a finite-by-Fuchsian group, where the Fuchsian group is not finite nor two-ended, and there is exactly one edge of $\Gamma$ which is incident to $v$ for each peripheral subgroup $K$ of $G(v)$ and this edge carries $K$. Thus a vertex cannot be both isolated and of finite-by-Fuchsian type.

Let $E_{1}$ denote the family of equivalence classes of all nontrivial almost invariant subsets of $G$ which are over a two-ended subgroup. It would seem simpler to directly consider the family of all such almost invariant subsets of $G$, rather than their equivalence classes. But there are situations where this causes problems. This is a minor point which the reader should ignore at a first reading. Our first canonical decomposition result is the following.

Theorem 2. If $G$ is a one-ended, finitely presented group, then there is a regular neighbourhood $\Gamma\left(E_{1}: G\right)$ of $E_{1}$ in $G$. Each $V_{0}$-vertex $v$ of $\Gamma\left(E_{1}: G\right)$ satisfies one of the following conditions:

(1) $v$ is isolated.

(2) $v$ is of finite-by-Fuchsian type.

(3) $G(v)$ is the full commensuriser $\mathrm{Comm}_{G}(H)$ for some two-ended subgroup $H$, such that $e(G, H) \geq 2$.

$\Gamma\left(E_{1}: G\right)$ consists of a single vertex if and only if $E_{1}$ is empty, or $G$ itself satisfies one of conditions (2) or (3) above.

We will denote $\Gamma\left(E_{1}: G\right)$ by $\Gamma_{1}$. Thus $\Gamma_{1}$ is a finite bipartite graph of groups decomposition of $G$ with the following properties. Any element of $E_{1}$ is enclosed by some $V_{0}$-vertex of $\Gamma_{1}$, and each $V_{0}$-vertex of $\Gamma_{1}$ encloses some element of $E_{1}$. In particular, any splitting of $G$ over a two-ended subgroup is enclosed by some $V_{0}$-vertex of $\Gamma_{1}$. If $\sigma$ is a splitting of $G$, and if $\sigma$ has intersection number zero with every element of $E_{1}$, then $\sigma$ is enclosed by a $V_{1}$-vertex of $\Gamma_{1}$. Our uniqueness result for regular neighbourhoods implies that this decomposition is unique. 
The uniqueness of $\Gamma_{1}$ has further implications. As $E_{1}$ is obviously invariant under automorphisms of $G$, it follows that the same holds for $\Gamma_{1}$. This is one of the main features of our work. Previously the only such result was for the special case when $G$ is word hyperbolic.

A remarkable feature of the decomposition $\Gamma_{1}$ is that $V_{0}$-vertex groups of type (3) need not be finitely generated. This does not contradict the comments after Theorem 1 as the family $E_{1}$ may be infinite.

It is possible to obtain the decompositions of previous authors from $\Gamma_{1}$ by splitting $\Gamma_{1}$ at the $V_{0}$-vertices of commensuriser type. Doing this yields decompositions in which all the vertex groups are finitely generated, but these decompositions are no longer unique in general.

When $G$ is the fundamental group of a Haken manifold $M$, the $V_{0}$-vertices of $\Gamma_{1}$ essentially correspond to the peripheral components of the characteristic submanifold $V(M)$ of $M$. In particular, the decomposition of $G$ given by $\Gamma_{1}$ essentially corresponds to the full characteristic submanifold when $M$ is atoroidal. The isolated $V_{0}$-vertices correspond to components of $V(M)$ of the form $A \times I$, where $A$ denotes an annulus, the $V_{0}$-vertices of finite-by-Fuchsian type correspond to $I$ bundle components of $V(M)$, and the $V_{0}$-vertices of commensuriser type correspond to peripheral Seifert fibre space components of $V(M)$.

Now we discuss generalisations of the above result. Let $E_{k}$ denote the collection of equivalence classes of all the nontrivial almost invariant subsets of $G$ over virtually polycyclic (VPC) subgroups of $G$ of Hirsch length $k$. (For brevity, we will refer to the length rather than the Hirsch length of a VPC group throughout the rest of this paper.) Note that $E_{0}$ is empty if $G$ is one-ended. We will need the following generalisation of the idea of a vertex of Fuchsian type. A vertex $v$ of a graph of groups $\Gamma$ is of VPC-by-Fuchsian type if $G(v)$ is a VPC-by-Fuchsian group, where the Fuchsian group is not finite nor two-ended, and there is exactly one edge of $\Gamma$ which is incident to $v$ for each peripheral subgroup $K$ of $G(v)$ and this edge carries $K$. If the length of the normal VPC subgroup of $G(v)$ is $k$, we will say that $G(v)$ is of length $k$. Note that if $G=G(v)$, then the Fuchsian quotient group corresponds to a closed orbifold.

Our first generalisation is the following result.

Theorem 3. If $G$ is a one-ended, finitely presented group which does not split over $V P C$ groups of length $<n$, then there is a regular neighbourhood $\Gamma\left(E_{n}: G\right)$ of $E_{n}$ in $G$. Each $V_{0}$-vertex $v$ of $\Gamma\left(E_{n}: G\right)$ satisfies one of the following conditions:

(1) $v$ is isolated, so that $G(v)$ is VPC of length $n$.

(2) $G(v)$ is of VPC-by-Fuchsian type of length $(n-1)$.

(3) $G(v)$ is the full commensuriser $\operatorname{Comm}_{G}(H)$ for some VPC subgroup $H$ of length $n$, such that $e(G, H) \geq 2$.

$\Gamma\left(E_{n}: G\right)$ consists of a single vertex if and only if $E_{n}$ is empty, or $G$ itself satisfies one of the above three conditions.

We denote $\Gamma\left(E_{n}: G\right)$ by $\Gamma_{n}$. For $n=2$, the special case of this result when $G$ is the fundamental group of a closed Haken 3-manifold $M$ essentially yields the topological JSJ-decomposition of $M$. For $n \geq 2$, the special case of this result when $G$ is a $P D(n+1)$-group, i.e., a Poincaré duality group of dimension $n+1$, recovers the results of Kropholler in [11]. Our results are slightly more general because they apply to all Poincaré duality groups, whereas Kropholler's results 
apply only to Poincaré duality groups such that any VPC subgroup has finitely generated centraliser. An example due to Mess [12] shows that this condition is not always satisfied. It should be noted that if a group $G$ has some special structure, then the vertices of $\Gamma_{n}$ may also satisfy special conditions. For example, when $G$ is a $P D(n+1)$-group, the $V_{0}$-vertices of $\Gamma_{n}$ of type $(3)$ must themselves be $P D(n)$ groups. In particular, every $V_{0}$-vertex group must be finitely generated. This last condition holds also if $G$ is a $\operatorname{Cat}(0)$-group.

In our next generalisation, we construct an algebraic analogue of the full characteristic submanifold of a 3 -manifold. Starting with the decomposition $\Gamma_{1}$ of a group $G$, we consider how to enclose elements of $E_{2}$. The closest analogue to the topology is obtained by considering only those elements of $E_{2}$ which have intersection number zero with every element of $E_{1}$. These are called 1 -canonical. We let $E_{1,2}$ denote the union of $E_{1}$ together with the 1-canonical elements of $E_{2}$. The result which we obtain is the following.

Theorem 4. If $G$ is a one-ended, finitely presented group, then there is a regular neighbourhood $\Gamma_{1,2}=\Gamma\left(E_{1,2}: G\right)$ of $E_{1,2}$ in $G$.

Each $V_{0}$-vertex $v$ of $\Gamma_{1,2}$ satisfies one of the following conditions:

(1) $v$ is isolated, so that $G(v)$ is VPC of length 1 or 2.

(2) $v$ is of VPC-by-Fuchsian type of length 0 or 1.

(3) $G(v)$ is the full commensuriser $\operatorname{Comm}_{G}(H)$ for some VPC subgroup $H$ of length 1 or 2 , such that $e(G, H) \geq 2$.

$\Gamma_{1,2}$ consists of a single vertex if and only if $E_{1,2}$ is empty, or $G$ itself satisfies one of the above three conditions.

The graph of groups structure $\Gamma_{1,2}$ for $G$ is a refinement of $\Gamma_{1}$ which can be obtained from $\Gamma_{1}$ by adding new vertices which enclose all the 1-canonical elements of $E_{2}$. In the case when $G$ is the fundamental group of a Haken 3-manifold $M$, we show that this essentially corresponds to the topological JSJ-decomposition of $M$. This uses our work in [17]. It may seem unsatisfactory that we do not find a decomposition of $G$ with vertex groups which enclose all elements of $E_{1}$ and $E_{2}$, but our work in [17] shows that if such a decomposition exists, it cannot be a refinement of $\Gamma_{1}$. Thus there may be almost invariant subsets of $G$ which are over VPC subgroups of length 2 but are not enclosed by any $V_{0}$-vertex of $\Gamma_{1,2}$. However, we show that the stabiliser of such an almost invariant subset of $G$ has a subgroup of finite index which is conjugate into a $V_{0}$-vertex of $\Gamma_{1,2}$.

If $G$ does not split over any VPC subgroups of length less than $n$, then we start from the decomposition $\Gamma_{n}$. We say that an element of $E_{k}$ is $n$-canonical if it has intersection number zero with every element of $E_{i}$, for $i \leq n$. As in the case when $n=1$, we show that $\Gamma_{n}$ can be refined to a graph of groups structure $\Gamma_{n, n+1}$ for $G$ by adding new vertices which enclose all the $n$-canonical elements of $E_{n+1}$.

Finally we consider the natural question of whether one can continue in the same way. We would like to refine $\Gamma_{1,2}$ to $\Gamma_{1,2,3}$ by adding new vertices which enclose all the 2-canonical elements of $E_{3}$, but there are simple examples to show that in general such a refinement does not exist. However, if we restrict attention to virtually abelian subgroups of $G$, such a refinement always exists, and the process can be continued for any finite number of stages. Let $A_{k}$ denote the collection of equivalence classes of all the nontrivial almost invariant subsets of $G$ over virtually abelian (VA) subgroups of $G$ of rank $k$. Let $B_{k}$ denote those elements of $A_{k}$ which 
have intersection number zero with every element of $A_{i}$, for $i<k$, and let $E_{1,2, \ldots, n}$ denote the union of $A_{1}$ and of $B_{k}, 2 \leq k \leq n$. Here is the result we obtain.

Theorem 5. If $G$ is a one-ended, finitely presented group, then there is a regular neighbourhood $\Gamma_{1,2, \ldots, n}=\Gamma\left(E_{1,2, \ldots, n}: G\right)$ of $E_{1,2, \ldots, n}$ in $G$.

Each $V_{0}$-vertex $v$ of $\Gamma_{1,2, \ldots, n}$ satisfies one of the following conditions:

(1) $v$ is isolated, so that $G(v)$ is VA of rank at most $n$.

(2) $G(v)$ is of VA-by-Fuchsian type of rank $k<n$.

(3) $G(v)$ is the full commensuriser $\mathrm{Comm}_{G}(H)$ for some VA subgroup $H$ of rank at most $n$, such that $e(G, H) \geq 2$.

$\Gamma_{1,2, \ldots, n}$ consists of a single vertex if and only if $E_{1,2, \ldots, n}$ is empty, or $G$ itself satisfies one of the above three conditions.

Acknowledgments: The first author gratefully acknowledges the support of the Hebrew University, the University of Melbourne, and the University of Warwick. His visit to Warwick was supported by the EPSRC. Both authors acknowledge the support of the Universite Paul Sabatier in Toulouse. They were supported by the ARC while in Melbourne, and by the CNRS while in Toulouse.

\section{REFERENCES}

[1] M. Bestvina and M. Feighn, Bounding the complexity of simplicial actions on trees, Invent. Math. 103 (1991), 145-186. MR 92c:20044

[2] B. H. Bowditch, Cut points and canonical splittings of hyperbolic groups, Acta Math. 180 (1998), 145-186. MR 99g:20069

[3] B. H. Bowditch, Splittings of finitely generated groups over two-ended subgroups, Trans. Amer. Math. Soc. 354 (2002), no. 3, 1049-1078.

[4] M. J. Dunwoody and M. Roller, Splitting groups over polycyclic-by-finite subgroups, Bull. London Math. Soc. 25 (1993), 29-36. MR 93h:20029

[5] M. J. Dunwoody and M. E. Sageev, JSJ-splittings for finitely presented groups over slender groups, Invent. Math. 135 (1999), 25-44. MR 2000b:20050

[6] M. J. Dunwoody and E. Swenson, The Algebraic Torus Theorem, Invent. Math. 140 (2000), 605-637. MR 2001d:20039

[7] M. Freedman, J. Hass, and G. P. Scott, Least area incompressible surfaces in 3-manifolds, Invent. Math. 71 (1983), 609-642. MR 85e:57012

[8] K. Fujiwara and P. Papasoglu, JSJ-decompositions for finitely presented groups and complexes of groups, Preprint (1997).

[9] W. Jaco and P. Shalen, Seifert fibered spaces in 3-manifolds, Memoirs of Amer. Math. Soc. vol. 21, Number 220 (1979). MR 81c:57010

[10] K. Johannson, Homotopy equivalences of 3-manifolds with boundary, Lecture Notes in Mathematics, vol. 761, Springer-Verlag, 1979. MR 82c:57005

[11] P. H. Kropholler, An analogue of the torus decomposition theorem for certain Poincaré duality groups, Proc. London. Math. Soc. (3) 60 (1990), 503-529. MR 91g:20079

[12] G. Mess, Examples of Poincaré duality groups, Proc. Amer. Math. Soc. 110 (1990), no. 4, 1145-1146. MR 91c:20075

[13] E. Rips and Z. Sela, Cyclic splittings of finitely presented groups and canonical JSJdecomposition, Annals of Mathematics 146 (1997), 53-109. MR 98m:20044]

[14] P. Scott, The Symmetry of Intersection Numbers in Group Theory, Geometry and Topology 2 (1998), 11-29, Correction (ibid) (1998). MR 99k:20076a MR 99k:20076b

[15] P. Scott and G. A. Swarup, Splittings of groups and intersection numbers, Geometry and Topology 4 (2000), 179-218. MR 2001h:20032

[16] P. Scott and G. A. Swarup, An Algebraic Annulus Theorem, Pacific Journal of Math. 196 (2000), 461-506. MR 2001k:20090

[17] P. Scott and G. A. Swarup, Canonical splittings of groups and 3-manifolds, Trans. Amer. Math. Soc. 353 (2001), 4973-5001. MR 2002f:57002 
[18] Z. Sela, Structure and rigidity in (Gromov) hyperbolic groups and rank 1 Lie groups, Geometric and Functional Anal. 7 (1997), 561-593. MR 98j:20044

[19] F. Waldhausen, On the determination of some bounded 3-manifolds by their fundamental groups alone, Proc. of the International Sympo. on Topology and Its Applications, HercegNovi, Yugoslavia, Beograd, 1969, 331-332. MR 42:2416

Mathematics Department, University of Michigan, Ann Arbor, Mi 48109, USA

E-mail address: pscott@umich.edu

Department of Mathematics and Statistics, University of Melbourne, Victoria 3010 , Australia

E-mail address: gadde@ms.unimelb.edu.au 